\title{
Analizando la pobreza desde los pobres: propuesta de un marco teórico integrador
}

Federico Ricardo Harrison Alvergue ${ }^{1}$

Recibido en diciembre de 2015, aceptado en marzo de 2016

\begin{abstract}
Resumen
El presente trabajo representa un esfuerzo por construir un marco teórico que permita analizar la pobreza a partir de las dinámicas económicas, culturales y sociales en las que viven quienes se encuentran en esa condición. Para llevar a cabo esta tarea se integraron conceptos centrales de la Escuela Austriaca de Economía (el problema de coordinación), de la Nueva Economía Institucional (el impacto de las instituciones informales) y de la Nueva Sociología Económica (las redes sociales y la noción de embeddedness de la actividad económica). El resultado es un modelo de análisis que permite abordar la pobreza desde una perspectiva diferente de la que tradicionalmente se ha venido empleando en el ámbito de la economía política.
\end{abstract}

\section{Palabras claves}

Pobreza, Escuela Austríaca de Economía, dinámicas económicas, dinámicas culturales, dinámicas sociales, instituciones informales.

\section{Abstract}

This paper represents an effort to build a theoretical framework to analyze poverty from economic, cultural and social dynamics in living those in that condition. To accomplish this task central concepts of the Austrian School of Economics (the problem of coordination) of the New Institutional Economics (the impact of informal institutions) and the New Economic Sociology (social networks and the concept of integrated embeddedness of economic activity). The result is an analytical model that can address poverty from a different perspective than has traditionally been used in the field of political economy.

\section{Keywords}

Poverty, Austrian School of Economic, economy dynamic, cultural dynamic, social dynamic, informal institutions.

1. Maestro en Comunicaciones, Director Académico de la Escuela de Comunicación Mónica Herrera, El Salvador. E-mail: falvergue@monicaherrera.com 


\section{Introducción}

Uno de los temas centrales en documentos, discursos y programas políticos es la pobreza, al grado que resulta casi imposible concebir una oferta electoral o un plan de gobierno en el que no ocupe un lugar predominante. Sin embargo, este énfasis en el fenómeno de la pobreza y la preocupación que despierta en amplios sectores de la sociedad ${ }^{2}$ contrasta, por ejemplo, con el optimismo de instituciones como el Banco Mundial, que destaca que en los últimos veinte años la pobreza se ha reducido en más de la mitad (del 43.1 al 20.6\%) en todo el mundo (The World Bank, 2014). De igual forma, evidencia empírica que señala la disminución de la pobreza gracias a la globalización y el crecimiento económico (por ejemplo, Dollar \& Kraay, 2004) desentona con el éxito internacional de libros como Capital in the Twenty-First Century (2014) de Thomas Piketty y Arthur Goldhammer, que plantea un escenario bastante más pesimista.

¿A qué obedece esta falta de consenso respecto de la pobreza? ¿Por qué preocupa cada vez más a un mundo en el que cada día que pasa parece haber menos pobres? Estas preguntas pueden responderse de diferentes maneras: es posible que a políticos y académicos, dados sus sistemas de incentivos, les interese mantener el debate inconcluso; o puede ser que el ritmo al que se reduce la pobreza resulte insuficiente en un entorno en el que la tecnología avanza a pasos agigantados; también puede suponerse que, a pesar de los avances en ciencias como la economía o la sociología, es muy poco lo que se comprende sobre la pobreza y las dinámicas sociales y culturales de quienes viven en esta condición.

Este trabajo parte de esa última suposición y se desarrolla desde de la premisa que el debate sobre la pobreza, al distanciarse cada vez más de los pobres, es decir, de las personas que viven en esa condición, difícilmente puede conducir a soluciones reales y sostenibles. Mientras no exista una comprensión profunda y multidisciplinaria del fenómeno de la pobreza, el destino de quienes la sufren se seguirá definiendo en el terreno de juego de los combates ideológicos y las negociaciones políticas. En este sentido, lo que aquí se pretende es sentar las bases de un marco teórico de la pobreza que tenga como punto de partida los individuos y que permita identificar los factores que inciden en que esta condición se perpetúe, o bien, que la persona puede superarla.

Este marco teórico busca responder a la siguiente pregunta: ¿de qué manera puede estructurarse un programa de investigación que permita estudiar de manera sistemática y progresiva, desde diversas áreas de conocimiento, la condición de pobreza en la que aún viven cientos de millones de personas? En la construcción de este marco teórico se hace un esfuerzo explícito por

2. A la larga lista de organizaciones no gubernamentales dedicadas al estudio y combate de la pobreza, pueden agregarse las iniciativas formales de prestigiosas universidades alrededor del mundo, por ejemplo, el Stanford Center on Poverty and Inequality, el Abdul Latif Jameel Poverty Action Lab del MIT o el Oxford Poverty \& Human Development Institute. 
integrar los aportes de la economía con los de otras disciplinas, como la sociología, con el propósito de desarrollar una mirada más amplia y completa que las tradicionales. En la sección II se presenta una revisión del abordaje de la pobreza que se hace en la economía como ciencia y sus aplicaciones a la política. En la sección III se argumenta que la pobreza es el resultado de un problema de coordinación entre los actores sociales. La sección IV ofrece una comprensión de dicho problema desde la perspectiva de la Nueva Economía Institucional, la cual se complementa con los aportes teóricos de la Nueva Sociología Económica expuestos en la sección V. En la sección VI se sintetiza un modelo de análisis utilizando los principios de la metodología de Lakatos para el desarrollo de programas de investigación científica para, finalmente, presentar las conclusiones y las rutas de investigación abiertas.

\section{La Pobreza y su Pobre Discusión}

The history of poverty is almost the history of mankind. The ancient writers have left us few specific accounts of it.

They took it for granted. Poverty was the normal lot.

(Hazlitt, 1996, pág. 13)

\section{En busca de una definición}

Parece lógico pensar que la economía es la ciencia llamada a liderar el debate y el análisis de la pobreza, dado que su objeto central de estudio suele asociarse con las decisiones que conducen a la generación de la riqueza; visto de otra manera, con el camino para reducir la pobreza. Sin embargo, el abordaje de los economistas sobre la pobreza suele ser, en términos generales, ambiguo y abstracto.

La ambigüedad inicia en su definición misma. El autor del epígrafe con el que inicia este capítulo subraya los vacíos que se presentan en la discusión de pobreza:

Of the thousands of books and articles on the subject that have appeared over the last two centuries, it is astonishing how few have troubled to ask this question [precisely what is poverty?]. Their writers have taken it for granted that both they and their readers know precisely what is being discussed. Yet popularly the term is very vague. It is always employed in a relative rather than an absolute sense. (Hazlitt, 1996, pág. 31).

Las diferentes formas de conceptualizar y comprender la pobreza en la sociedad contemporánea tienen su origen en las metodologías que han sido 
utilizadas para medirla (Niemietz, 2011). Las primeras mediciones de pobreza, desarrolladas por Charles Booth y Benjamin Seebohm Rowtree a finales del siglo XIX en Inglaterra, se centraban en la satisfacción de necesidades físicas como nutrición, abrigo y vestuario. Para estos investigadores una persona pobre era quien no podía cubrir un mínimo de estas necesidades. Varias décadas después, a mediados del siglo XX, cuando se consideraba que el fenómeno había sido erradicado (al menos en Inglaterra), se produce un redescubrimiento de la pobreza a través de la conceptualización y medición de la pobreza relativa. En este caso, la línea de pobreza se fija como una fracción del ingreso promedio de las personas. Posteriormente se han desarrollado otras mediciones, como la pobreza subjetiva y la privación material. Cada una de estas definiciones tiene consecuencias diversas en el diseño de políticas, por lo que la medición de la pobreza no debe ser vista como un dato puramente técnico (Niemietz, 2011).

Los dos caminos predominantes de definirla - de forma absoluta y relativason expuestos en manuales generales de economía (e.g. Baumol \& Blinder, 1994; Samuelson \& Nordhaus, 1999; Tucker, 2002), en los que se percibe una inclinación hacia la forma relativa de entenderla. De hecho, la norma general en los libros de texto parece ser la de discutir la pobreza precediendo o sucediendo la presentación del tema de desigualdad y/o distribución del ingreso. ${ }^{3}$ No obstante, como lo señala Krause, se trata de cosas diferentes: "la discusión sobre la pobreza, su medición y las políticas que de eso se desprenden han girado hacia concepciones 'relativas' que miden la distribución, no la falta de medios para satisfacer ciertas necesidades básicas” (Krause, 2013, pos. 289).

\section{Una discusión cada vez más abstracta}

La mirada de la pobreza como un fenómeno esencialmente relativo ha permeado el campo de la economía política, donde su vinculación con la "desigualdad del ingreso" o con la "injusta distribución de la riqueza y la renta" es materia común. A modo de ejemplo, pueden revisarse trabajos de amplia difusión internacional como el de Joseph Stiglitz, que vincula la pobreza y la desigualdad con el proceso de globalización (Stiglitz, 2006) ${ }^{4}$ o el Informe sobre Desarrollo Humano 2013 del Programa de las Naciones Unidas para el Desarrollo (PNUD), que sugiere que "no será posible sostener el progreso en desarrollo humano a menos que se ponga en primer plano el debate político sobre desigualdad y destrucción ambiental” (PNUD, 2013a, págs. 2-3).

Esta perspectiva acerca del fenómeno de la pobreza presenta un problema: confina la discusión al mundo de las generalizaciones, las abstracciones conceptuales, los datos demográficos y los cálculos estadísticos. El resultado de

3. Además de los manuales ya citados pueden agregarse Mandel, 2009; Mankiw, 2002; y Parkin, 2004. 4. Resulta ilustrativo de esta situación que en el índice onomástico de texto de Stiglitz (2006), se lee bajo el tema de pobreza "see also income, income inequality". 
un abordaje de este tipo es que el debate sobre la pobreza ha pasado a centrarse en los aspectos metodológicos de su medición, en discusiones conceptuales y en la validez de la fuente de los datos utilizados (Ravallion, 2003), más que en las dinámicas culturales, sociales y particulares que mantiene en esa condición a las personas. ${ }^{5}$ En el afán por encontrar explicaciones y análisis “objetivos" se cae en un problema señalado hace casi siete décadas por Friedrich A. Hayek: “this view which regards social collectivities such as 'society' or the 'state', or any particular social institution or phenomenon, as in any sense more objective than the intelligible actions of the individuals is sheer illusion" (Hayek, 1948b, pág. 69). Un ejemplo reciente es la discusión alrededor del trabajo de Thomas Piketty (2014) y las metodologías estadísticas utilizadas su investigación. ${ }^{6}$

El problema de fondo que aquí se quiere enfatizar ha sido señalado con claridad por Kristian Niemietz: "[t]he language of the poverty debate seems to be designated to obscure rather than illuminate a proper understanding of the issue" (Niemietz, 2011, págs. 29-30).

La mayor (y peor) consecuencia de esta mirada positivista de la pobreza es que el fenómeno pasa a comprenderse como un problema técnico que, por lo tanto, pareciera requerir de soluciones "técnicas". Los orígenes, formas y consecuencias de esta aproximación tecnócrata son expuestos por William Easterly:

The conventional approach to economic development, to making poor countries rich, is based on a technocratic illusion: the belief that poverty is a purely technical problem amenable to such technical solutions as fertilizers, antibiotics, or nutritional supplements... The sleight of hand that focuses attention on technical solutions while covering up violations of the rights of real people is the moral tragedy of development today. (Easterly, 2013, pág. 6)

El autor presenta una crítica del enfoque desarrollista en la economía (impulsado por los países desarrollados de Occidente a partir del final de la Primera Guerra Mundial mediante organismos como el Banco Mundial y el Fondo Monetario Internacional) que sugiere que es posible diseñar el camino hacia el progreso bajo una aproximación "científica”. Su crítica se sustenta en que la ejecución de la planificación centralizada del desarrollo requiere de regímenes autocráticos que, en mayor o menor medida, anulan derechos y libertades individuales. Para Easterly, la reducción de la pobreza requiere, en primer lugar, del reconocimiento de la historia y las características culturales de cada sociedad; en segundo lugar, del respeto de los derechos fundamentales de sus pobladores; y en tercer

5. Por supuesto hay excepciones, como el estudio Voices of the Poor. Can Anyone Hear Us? (Narayan, Patel, Schafft, Rademacher, \& Koch-Schulte, 2000).

6. Puede verse una crítica de Chris Gilles (2014) en el "Financial Times", y la defensa de Paul Krugman (2014) en su blog de "The New York Times" y de Paul Mason (2014) en "The Guardian". 
lugar, y como consecuencia de los condicionantes anteriores, de la formulación de soluciones e instituciones que surjan desde las personas involucradas (en clave hayekiana, órdenes espontáneos). Este enfoque, evidentemente, no es compatible con la mirada "objetiva" de la pobreza.

En términos teóricos, es posible aseverar que el abordaje tecnócrata que Easterly critica es el resultado de la tradición neoclásica o mainstream de la economía y que, por lo tanto, corrientes de pensamiento que tienen su base epistemológica en el individualismo metodológico están en condiciones de ofrecer mejores explicaciones del fenómeno de la pobreza. En este sentido, la Escuela Austriaca de Economía (EAE) se antoja como una candidata ${ }^{7}$, sin embargo, su abordaje de la pobreza se limita a verla únicamente como una condición natural que antecede al desarrollo del capitalismo y que se supera automáticamente con el desarrollo de éste. Al menos en su corriente teórica más difundida, la EAE no dedica atención especial al fenómeno de la pobreza. Por ejemplo, Ludwig von Mises la aborda como el resultado ineludible de la falta de aplicación del capitalismo de libre mercado (Mises, [1949] 1996, págs. 835-836). Por su parte, Hayek también se refiere a la pobreza como un fenómeno relativo y una condición previa al capitalismo que se supera gracias a los procesos productivos y de acumulación del capital que éste facilita (Hayek, 1978, pág. 44). En Murray Rothbard ([1962] 2001) las referencias a la pobreza son aún menores, limitándose a un crítica a los programas de ayuda a los pobres de parte del Estado (pág. 818) y a una observación metodológica al trabajo de Galbraith (pág. 840).

\section{Un camino para avanzar en la discusión}

Como se señala en los párrafos anteriores, la discusión sobre la pobreza ha transitado hacia debates estadísticos, cada vez más alejados de las condiciones y dinámicas particulares de las personas que viven en esa condición. El resultado de esta aproximación es una concepción de la pobreza como un problema técnico que puede estudiarse de manera “objetiva” y "científica” para luego resolverse con soluciones técnicas y replicables.

Esta mirada ha conducido a la construcción de una red cada vez más extensa de organismos multilaterales de cooperación para el desarrollo y de oficinas públicas cuyo propósito explicito es el de erradicar la pobreza. El resultado, sin embargo, es el ya señalado por la escuela del Public Choice y que Christopher Coyne y Peter Boettke apuntan en su artículo The Role of the Economist in Economic Development:

7. En The Tyranny of Experts. Economics, Dictators, and the Forgotten Rights of the Poor (2013), Easterly cita de manera extensa The Road to Serfdom del austriaco F. A. Hayek. 
The... reason for the persistence of the conventional role of the economist is the perverse incentives faced by both those both in the development community and those in government in developing countries. Although the stated goal of the development community is to eradicate poverty and social ills, there is a strong incentive for those involved to fail in achieving this goal. Indeed, if the ultimate goal were in fact accomplished, those in the international development community would eradicate their own source of employment. (Coyne \& Boettke, 2006, pág. 62)

Este señalamiento sugiere una reconsideración del rol del economista en el estudio de la pobreza, así como de la forma en la que ésta debe abordarse. Los autores lo presentan de esta manera en sus conclusiones:

Our reconsideration of the role of the economist in economic development concluded that there is a significant role for the economist to play in this area. The discipline of economics provides the economist with the tools to be a student of the economic system. He is suited to understand the interplay of both formal and informal institutions and their impact on economic activity... Truly understanding the plight of underdeveloped nations requires a complete comprehension of both formal and informal institutions. (Coyne \& Boettke, 2006, pág. 65) [cursivas en el original]

El presente trabajo se fundamenta en la idea que la pobreza puede y debe estudiarse a partir de los marcos institucionales que prevalecen en una sociedad, poniendo especial énfasis en las instituciones informales. Pero antes de proceder al análisis de las instituciones, se retomará una idea central en la tradición austriaca: el problema de coordinación.

\section{La Pobreza como un Problema de Coordinación}

To assume all the knowledge to be given to a single mind in the same manner in which we assume it to be given to us as the explaining economists is to assume the problem away and to disregard everything that is important and significant in the real world. (Hayek, 1945, pág. 530)

La mirada tecnócrata y su metodología para abordar la pobreza -tanto sus causas como sus posibles soluciones- es consecuente con el paradigma neoclásico en la economía: se asume como un problema de (in)eficiencia en la asignación de los recursos, (des)equilibrio y maximización de utilidades dentro de un mundo de decisiones racionales en el que es posible acceder a toda la información 
necesaria. Steven Horwitz sintetiza esta idea de la siguiente manera:

For neoclassical economics, the humans who populate its model are assumed to know a great deal about the structure of their problem situation. They know their own utility or production function, they know all of the relevant market prices, and they know all else that is relevant in order for them to maximize utility or profits. Maximization is simply solving the problem that is implicit in the data. Agents know the ends they wish to pursue and they know what means are at their disposal. The optimal solution is automatic; there is no meaningful choice. (Horwitz, 2000, pág. 29)

Alejarse de esta perspectiva con miras a abordar la condición de los pobres como un problema de seres humanos y no de estadísticas exige plantearlo desde un paradigma diferente. En los términos de Christopher Coyne (2010), esto significa transitar del paradigma de la asignación (allocation) al del intercambio (exchange). Para ello es menester concebir el problema económico de la forma en la que Hayek lo proponía: como un problema de coordinación (O’Driscoll, 1977) inserto en lo que, para él, representa la principal interrogante de las ciencias sociales:

How can the combination of fragments of knowledge existing in different minds bring about results which, if they were to be brought about deliberately, would require a knowledge on the part of the directing mind which no single person can possess? (Hayek, 1948a, pág. 54)

Para abordar el fenómeno de la pobreza desde esta perspectiva, la atención se debe centrar en las acciones de los actores involucrados, concretamente en las maneras en las que se relacionan, comunican, aprenden mutuamente y coordinan sus actividades, así como en el marco institucional que surge para facilitarlo. Las discusiones sobre las estadísticas y la desigualdad deben pasar a un plano complementario, alejado del núcleo del debate.

Aunque Hayek empleaba la noción de coordinación fundamentalmente para explicar el camino hacia la condición de equilibrio (O’Driscoll, 1977) y para discutir la generación de prosperidad (Hayek, 1945, 1948a), es posible extrapolarlo y utilizarlo como un marco de referencia para comprender el fenómeno de la pobreza. De hecho, la tendencia a la coordinación de la acción humana es parte del núcleo central del programa de investigación de la Escuela Austriaca (Rizzo, 1982). De ahí que una premisa del presente trabajo es que la pobreza debe ser comprendida como el resultado de una coordinación deficiente entre los individuos, o bien de una falta de coordinación. Como lo señalan Narayan et al. (2000), la capacidad de los pobres para organizarse (i.e. coordinarse) equivale a su capital social, el cual constituye su mayor activo. 
Continuando con la tradición de la EAE, esta vez en los términos de Israel Kirzner (1997), podría hablarse de una ausencia de descubrimiento empresarial ${ }^{8}$ producto de la falta de marcos institucionales adecuados. Las condiciones institucionales que subyacen a esta mala coordinación - $\mathrm{o}$ a la ausencia de coordinación- es uno de los temas centrales del presente trabajo.

En la siguiente sección se profundizará en el tema de las instituciones, pero antes de avanzar se hace necesario proponer una definición de pobreza que sintetice lo expuesto hasta este momento y permita avanzar en la discusión: la pobreza es la condición de escasez material que resulta de un deficiente proceso de coordinación social.

\section{El Marco Institucional de la Pobreza}

But there also are certain superindividual schemes of thought, namely, institutions, to which the schemes of thought of the first order, the plans, must be oriented, and which serve therefore, to some extent, the coordination of individual plans. (Lachmann, 1977, pág. 62)

Si se asume que la condición de pobreza es el resultado de un deficiente proceso de coordinación entre los miembros de una sociedad, se hace necesario escudriñar las características y condicionantes de los engranajes que facilitan -o dificultan- la interacción y coordinación: las instituciones. Este rol de las instituciones como facilitadores de la coordinación es precisamente lo que señala Ludwig Lachmann en el epígrafe con el que inicia esta sección. Otros autores también ponen énfasis en el rol coordinador de las instituciones, entre ellos, Peter Boettke en un estudio sobre la obra de Hayek: "[t]he social institutions that arise through the voluntary association of thousands of individuals serve to guide individuals in the process of mutual accommodation" (Boettke, 1990, pág. 69).

En los siguientes párrafos de esta sección se hace una breve exposición del origen y características de las instituciones desde el enfoque de la Nueva Economía Institucional, poniendo énfasis en su impacto en el desarrollo económico. Para finalizar, se destacarán los vacíos del enfoque institucional para explicar la condición de pobreza.

8. En términos reales no es posible hablar de una ausencia total de "descubrimiento empresarial", pero puede inferirse que, en el caso de personas en condición de pobreza, estos descubrimientos tienen un impacto limitado. 


\section{Generalidades sobre las instituciones y la perspectiva institucional en la economía}

De acuerdo con Geoffrey M. Hodgson (2006), el uso del término institución o instituciones en las ciencias sociales ha tenido una amplia expansión en los últimos años. En el caso de la economía, la sub-disciplina conocida como Nueva Economía Institucional (North, 2012) toma las instituciones como eje central para intentar comprender el funcionamiento del sistema económico alejándose de la tradición mainstream o neoclásica de aislar las variables. Desde esta perspectiva, cualquier sistema económico -ya sea que genere prosperidad o pobreza- descansa sobre un conjunto de instituciones. Por institución se entiende, en términos generales, el conjunto de normas o convenciones que facilitan la coordinación entre las personas. En palabras de Douglass North, "[institutions] are the humanly devised constraints that structure political, economic and social interaction... Throughout history, institutions have been devised by human beings to create order and reduce uncertainty in exchange" (North, 1991, pág. 97).

Dado que es posible confundir el significado de instituciones con el de reglas, Hodgson ofrece una definición que aclara esa posible confusión, al tiempo que intenta resolver lo que para él son ambigüedades en la mirada de North: "I define institutions as durable systems of established and embedded social rules that structure social interactions, rather than rules as such. In short, institutions are social rule-systems, not simply rules” (Hodgson, 2006, pág. 13) [cursivas en el original].

Al hablar de instituciones se debe distinguir entre dos tipos básicos: (a) las informales, que están constituidas por elementos culturales como por ejemplo los tabús, tradiciones o códigos de conducta, y (b) las formales, que son el resultado de hacerlas explícitas mediante las constituciones, las leyes o los derechos de propiedad, por citar algunos (North, 1990; North 1991). Éstas, junto con los mecanismos o características para "hacer cumplir" las normas (enforcement characteristics) constituyen la estructura institucional de una sociedad y definen su sistema de incentivos económicos (North, 2005).

El impacto de las instituciones en el desarrollo económico de un país y, por lo tanto, en sus niveles de pobreza, es abordado ampliamente por North, quien afirma que la historia del progreso o estancamiento económico de un país es consecuencia directa de su evolución institucional: "Institutions provide the incentive structure of an economy; as that structure evolves, it shapes the direction of economic change towards growth, stagnation, or decline" (North, 1991, pág. 97). Esta premisa da la pauta para abordar el fenómeno de la pobreza desde una perspectiva institucional. Dicho abordaje puede hacerse desde la óptica de las instituciones formales, desde las informales, o desde ambas (Williamson \& Mathers, 2011). 
No obstante, aunque en muchas ocasiones son pasadas por alto, las instituciones informales son las que posibilitan una mayor y mejor comprensión del progreso económico y de la pobreza. North lo plantea de esta forma al cierre de su ensayo "Institutions" (1991): "What is it about informal constraints that gives them such a pervasive influence upon the long-run character of economies?” (pág.111). Para responder a esta pregunta es menester abordar el papel preponderante que juegan la herencia cultural y las creencias en las estructuras institucionales. Continuando con North, "[w]e cannot see, feel, touch, or even measure institutions; they are constructs of the human mind" (North, 1990, pág.106).

\section{La relación causal entre cultura, instituciones y desempeño económico}

Tradicionalmente los economistas en la corriente mainstream se han rehusado a abordar el impacto de la cultura en los fenómenos económicos. Esto obedece, en parte, a la dificultad de reducir los fenómenos culturales a hipótesis capaces de ser testeadas empíricamente (Guiso, Sapienza, \& Zingales, 2006). Sin embargo, si se reconoce y acepta la influencia de las instituciones - principalmente las informales- en los fenómenos económicos y sociales en este caso, la pobreza-, no es posible obviar la influencia de la cultura. ${ }^{9}$ En esta línea de pensamiento, Peter Boettke (2001) enfatiza la necesidad de abordar cuestiones como la riqueza de las naciones y la desigualdad entre éstas desde una perspectiva multidisciplinaria, que integre la información empírica obtenida a través de la econometría tradicional con supuestos desarrollados en otras áreas de las ciencias sociales, por ejemplo, la etnografía. Contrario a lo que podría asumirse, adoptar la cultura como punto de partida del análisis económico no implica abandonar los supuestos del individualismo metodológico en función de un cierto determinismo cultural. Esta idea es relevante para este trabajo y requiere ser expuesta en los términos en que Boettke lo hace:

In making this turn, individual decision-making is not to be overlooked, but the context of decision moves to the center of analysis as opposed to the behavioral assumptions, and economic actions are recognized as embedded within an environment, rather than disembedded and abstract. In other words, there is no necessary reason to jettison the methodological individualism-the hallmark of rational-choice social science-but the methodological individualism practiced would not be the atomistic individualism of mainstream price theory. A more institutional individualism-which steers between both atomistic individualism and holistic institutionalism-emerges as the basic methodological starting point of analysis for political economy and social theory. (Boettke, 2001, pág. 249)

9. En el reciente "Informe sobre Desarrollo Humano El Salvador 2013" (2013b) el Programa de las Naciones Unidas para el Desarrollo (PNUD) pone un especial énfasis en los factores culturales que inciden en la condición de pobreza. 
Este autor sugiere que la tradición y la cultura configuran marcos institucionales compuestos por reglas explícitas e implícitas en los que se sustentan las decisiones y acciones individuales. Para él, la influencia de la cultura en las instituciones puede sintetizarse de la siguiente manera:

Institutions, following the New Institutionalist literature, are defined as those formal and informal rules which govern human behavior. Culture, as I am discussing the term, refers to those beliefs and ritual practices which legitimate institutions. In order to develop a theory of institutional change and acceptability, institutions must be linked to culture. (Boettke, 2001, pág. 254)

Por ejemplo, en tradiciones culturales en las que se concibe que las mujeres tienen un valor inferior que el de los hombres, surgen instituciones informales como la discriminación educativa o laboral, que tienen un fuerte impacto en el desarrollo económico y en la pobreza. ${ }^{10}$

De la relación causal entre cultura e instituciones surge la propuesta de Boettke de aplicar el teorema de la regresión (regression theorem) de Ludwig von Mises a los procesos de cambio institucional. Esto implica que las instituciones que persisten o se adhieren ${ }^{11}$ son aquellas con una raíz cultural susceptible de ser rastreada. Así, la institución del dinero solo es posible en un contexto cultural en el que ya hay una práctica de intercambio. De igual forma, el orden liberal y su correspondiente marco institucional se desarrollan a partir de bases culturales como el respeto a la propiedad privada que es consecuencia de reiteradas transacciones de intercambio en el mercado. Este teorema tiene fuertes implicaciones para el área de las políticas públicas y económicas, pues sugiere que cualquier nueva regla del juego, e.g. una legislación, únicamente será exitosa si se acopla a las normas culturales imperantes. Retomando el ejemplo de la discriminación a las mujeres, resulta difícil pensar que mediante legislaciones sea posible igualar sus derechos y condición de vida real. De acuerdo con Boettke, "[e]conomics may establish the properties of alternative rules, but culture and the imprint of history determine which rules can stick in certain environments” (Boettke, 2001, pág. 257) [cursivas en el original].

Investigaciones más recientes de Boettke, Coyne y Leeson (2008) les han llevado a proponer una taxonomía de instituciones que permite analizar su capacidad de adherirse (stickiness) a una sociedad. Esta taxonomía parte de dos tipos de orígenes opuestos. Por una parte, están las instituciones que emergen espontáneamente, desde dentro del grupo social y que son de carácter informal; los autores las llaman indigenously introduced endogenous institutions (IEN). Al

10. Ejemplos concretos de este caso pueden verse en Narayan et al. (2000).

11. Boettke utiliza el término en inglés stick para referirse a la cualidad de las instituciones que perduran o se insertan en la sociedad. 
otro extremo se encuentran las que son impuestas desde fuera y reciben el nombre de foreign introduced exogenous institution (FEX). Se identifica, además, un tipo de institución que representa una suerte de término medio entre las IEN y las FEX; se trata de las indigenously introduced exogenous institutions (IEX), que comprende a las que tienen un origen externo pero son impulsadas desde dentro. Para los autores, cualquier modificación institucional del tipo FEX o IEX que se pretenda instalar para, por ejemplo, potenciar el desarrollo económico, será adoptada por las personas únicamente si posee rasgos rastreables hasta una IEN, es decir, si se cumple el teorema de la regresión. En sus términos,

[s]uccessful institutional changes in developing parts of the world must have IEN institutions at their core. We place this claim at the center of the New Development Economics. To determine if any particular development-community proposal for institutional change meets this criteria, we suggest the following test: If the proposed change cannot be traced back to an IEN institution, it should not be attempted. (Boettke, Coyne, \& Leeson, 2008, pág. 344)

Aunque las IEN son necesarias para el desarrollo económico, no son suficientes. Además, debe destacarse que la adherencia de las instituciones de este tipo no presupone que tales instituciones sean beneficiosas o positivas: "institutional stickiness is not equivalent to institutional 'goodness' [...] In fact, many IEN institutions are themselves growth inhibitors" (Boettke et al, 2008, pág. 345) [cursivas en el original]. Poniendo un ejemplo extremo, una agrupación social en cuyo interior no existe el respeto a la vida e integridad de las personas (el irrespeto a la vida equivaldría a una IEN) no sería capaz de establecer los mecanismos de cooperación y coordinación necesarios para alcanzar la prosperidad, quedándose condenada a vivir en un perpetuo conflicto, a pesar de que en algún momento se dicte una ley que pretenda poner fin a esa práctica.

La necesidad de observar y respetar las instituciones informales endógenas es ampliamente discutida y respaldada por Easterly (2013), quien critica los supuestos de la Economía del Desarrollo y los esfuerzos de los organismos multilaterales por resolver el problema de la pobreza y el desarrollo a través de la imposición de instituciones formales estandarizadas. El autor se refiere a la práctica de la "pizarra en blanco" (blank slate), que supone la factibilidad y conveniencia de pasar por alto la herencia cultural de los "beneficiados". Estas políticas derivadas de un análisis estático en un ambiente altamente dinámico tienden a producir resultados negativos en el largo plazo (North, 2005).

Para precisar conceptualmente y con mayor nivel de detalle la relación causal entre la cultura, las instituciones y el desempeño económico, resulta de utilidad sintetizar el trabajo de North (2005). Para ello, se ha elaborado un diagrama (Figura 1) que se explica de la siguiente manera: a través de su historia, los grupos sociales producen de manera dinámica un conjunto 
de normas, valores e ideas que se transmiten de generación a generación; a este proceso se le denomina herencia cultural. Esta herencia cultural se manifiesta a través de un conjunto de artefactos, por ejemplo, un sistema de creencias, marcos institucionales (compuestos por instituciones informales y formales), herramientas, instrumentos y tecnologías. De este conjunto de artefactos destacan dos: el sistema de creencias y las instituciones. El primero corresponde a la representación interna de la herencia cultural; el segundo (las instituciones) son la manifestación externa de las creencias, es decir, aquello que es posible observar a través de comportamientos, rituales, códigos de conducta, etc. La relación causal entre ambos artefactos (destacada con una flecha más gruesa en el diagrama) es fundamental para comprender el origen de las instituciones, principalmente las informales. Tanto el sistema de creencias como las instituciones dan origen al stock (o cúmulo) de conocimientos que la sociedad emplea para hacer frente a sus desafíos y oportunidades. El capital humano -sea extenso o limitado- con el que cuenta una agrupación social es un producto directo del stock de conocimientos que haya conseguido construir a través de la concreción institucional de su sistema de creencias. Finalmente, el éxito o fracaso económico de una sociedad está determinado por la interacción de todos los artefactos antes destacados, pero fundamentalmente por las organizaciones que resultan de las instituciones y que producen un camino de dependencia (path dependence) difícil de modificar en el corto plazo. Esto respalda el escepticismo de Boettke, Coyne y Leeson (2008) acerca de las FEX como un mecanismo de desarrollo económico.

La línea argumentativa de North permite comprender por qué los autores neo institucionalistas defienden que el cambio institucional y el desarrollo económico tienen su origen en las creencias y la cultura, más que en los cambios deliberados efectuados en las instituciones formales.

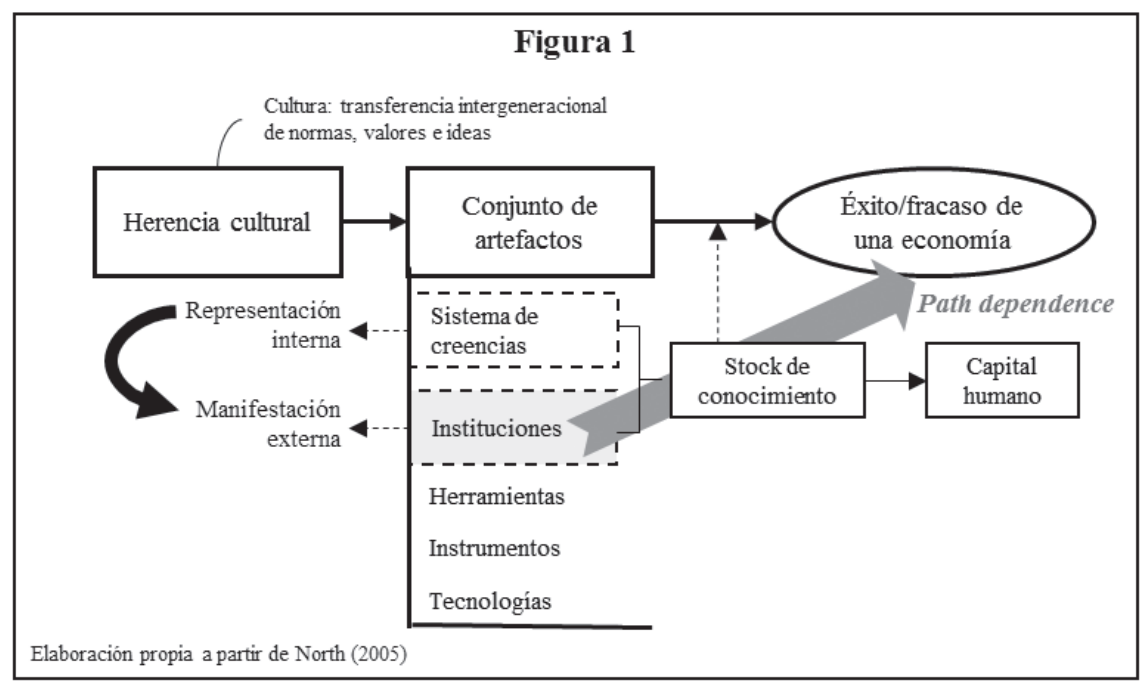


Estas elaboraciones teóricas sobre la influencia de la cultura y las instituciones informales en el desarrollo económico han sido puestas a prueba empíricamente. A continuación se presentan algunos ejemplos.

\section{Aproximaciones empíricas al impacto de la cultura en la economía}

Tanto Boettke et al (2008) como Williamson (2009) presentan evidencias empíricas de diversas regiones del mundo que refuerzan la tesis de que la instituciones informales o culturales influyen fuertemente en el desarrollo económico, así como que las instituciones formales son exitosas únicamente si se basan en instituciones informales previas. Las conclusiones de Claudia Williamson presentan implicaciones importantes para el diseño de políticas públicas que busquen disminuir la pobreza:

Informal constraints must exist in order to achieve economic success. It is possible to achieve positive returns from codifying these informal institutions. However, the likelihood of governments in developing countries having the knowledge and incentives to choose the right formal institutions is small. Most importantly, the idea that institutional arrangements are identifiable and transportable should now be taken with serious caution. (Williamson C. R., 2009, pág. 384)

Por su parte, Guiso, Sapienza y Zingales (2006) investigan el impacto de las creencias previas (prior beliefs) y los valores en el desempeño económico. Para esto se basan en dos variables exógenas que permiten evitar la influencia en dos vías o causalidad circular: la etnia y la religión. Su conclusión es que las hipótesis culturales pueden ser testeadas de formar rigurosa y que resultan ser relevantes en asuntos económicos como los niveles de ahorro en un país (Guiso, Sapienza, \& Zingales, 2006, págs. 45-46). Valentin Seidler analiza la forma y el grado de éxito con que instituciones formales son transferidas intencionadamente (transplant effect) en sociedades africanas, destacando el rol preponderante de las instituciones locales ya existentes para que las nuevas sean aceptadas (Seidler, 2014). Las investigaciones de Tabellini en diversas regiones de Europa cruzando datos históricos, macroeconómicos y resultados de las Encuestas Mundiales de Valores (World Value Surveys) indican que los factores culturales siguen siendo un enigma para explicar a cabalidad el desarrollo económico, sin embargo, el autor esgrime las siguientes conclusiones, que resultan importantes para el presente trabajo:

Two sets of cultural traits appear to be favorable to economic development. The first trait resembles what earlier studies have called "social capital," and is captured by the variables trust (having trust in other people) and respect (appreciating the virtue of having tolerance and respect for others in children). The second trait can be interpreted as confidence in individual, and is captured by the variable 
control (feeling in control of one's life) and, in a negative sense, by the variable obedience (appreciating obedience in one's own children). These cultural traits can influence economic development directly, or indirectly through the functioning of current institutions. (Tabellini, 2010, pág. 711) [cursivas en el original]

Estas conclusiones de Tabellini son afines a las conclusiones de las investigaciones de otros autores, algunos de ellos con trabajos dirigidos a audiencias no académicas. Por ejemplo, la idea de la confianza como un valor determinante para la prosperidad económica es desarrollada extensamente por Francis Fukuyama (1996), quien explica el desempeño económico y social de países como Alemania y Japón a partir del nivel de confianza que culturalmente poseen. Por su parte, las dimensiones culturales de control sobre la vida de uno mismo y la obediencia pueden verse en los estudios de Geert Hofstede sobre las diferencias culturales (Hofstede, 1999). Para este autor la cultura, en la forma de valores predominantes, es una condición necesaria pero no suficiente para explicar el desarrollo económico. De acuerdo con él, hay otras dos condiciones necesarias a las que ya se ha hecho alguna referencia en este trabajo: la existencia de un mercado (que puede entenderse como un espacio de coordinación social) y un contexto político (i.e. instituciones formales) que posibilite el desarrollo (Hofstede \& Harris Bond, 1988).

Coyne y Mathers (2011) llevan el análisis a una etapa previa a la conformación de valores y creencias al estudiar el impacto económico de los rituales. Aunque su investigación no se basa en evidencia empírica de primera mano, su abordaje del tema es sumamente valioso para los propósitos de este documento, especialmente porque -a través del papel que desempeñan los ritualesestablecen un vínculo entre la interacción de las personas y los procesos de coordinación que resultarán en el mayor o menor desarrollo económico y bienestar de los individuos:

[R]ituals can facilitate or prevent interactions, which will have real effects on economic outcomes for better or worse.... In other words, the willingness of someone to trade with a stranger ultimately requires that people respond in-kind and deliver on their responsibilities and agreements. Within this context, to the extent that rituals play a role in communicating trust and reciprocity, they can have a positive effect on economic outcomes. Likewise, to the extent that rituals constrain interactions by excluding outsiders, they can have a negative effect on development. (Coyne \& Mathers, 2011, pág. 75)

Esta referencia a la exclusión y la distinción simbólica entre “ellos" y "nosotros" que los autores plantean más adelante en su artículo será retomada en la próxima sección, pues permitirá complementar el enfoque institucional con miras a construir un marco teórico multidisciplinario de la pobreza. 
En este punto se hace necesario destacar que, así como muchos trabajos defienden la influencia predominante de las instituciones informales (culturales) en el desarrollo económico, también hay miradas que sugieren que el énfasis debe ponerse en las instituciones formales. Un buen ejemplo es Hernando de Soto, quien sostiene que una institución formal como los derechos de propiedad tendrá un impacto económico positivo a pesar de ser impuesta y no responder a un rasgo cultural. De hecho, critica directamente las explicaciones culturales del fenómeno del subdesarrollo:

Modestamente sugiero que antes de que cualquier gran señor que vive campana de vidrio adentro intente convencernos de que tener éxito en el capitalismo exige ciertos rasgos culturales, esperemos primero a ver qué sucederá cuando los países en vías de desarrollo y los que salen del comunismo establezcan sistemas de derechos de propiedad capaces de crear capital para todos... Los argumentos culturales irán siendo descartados a medida que se vayan dejando sentir los efectos de tener buenas instituciones políticas y leyes de propiedad. (de Soto, 2001, págs. 248-249)

\section{El vacío del enfoque institucional para comprender la pobreza}

Hasta este punto se han presentado los componentes iniciales para construir un marco teórico que permita analizar el fenómeno de la pobreza desde la situación de quienes viven en esa condición, alejado del enfoque tradicional centrado en datos, indicadores y estadísticas. Estas bases pueden resumirse en las siguientes tres ideas: (1) la condición de pobreza debe comprenderse como el resultado de un deficiente proceso de coordinación social; (2) el proceso de coordinación (o descoordinación) que conduce a la pobreza se basa en un marco institucional conformado por instituciones formales, informales y sus mecanismos de cumplimiento que definen los incentivos en un grupo social; (3) por sus raíces históricas y culturales, las instituciones informales son las que mayor influencia tienen en el desarrollo económico, pues de ellas depende el funcionamiento de las instituciones formales.

No obstante este enfoque institucional permite esbozar una comprensión general del fenómeno de la pobreza, hay que reconocer que el problema amerita un esfuerzo mayor por incorporar al análisis los elementos que anteceden a la formación de las instituciones informales. Solo de esta manera se estaría partiendo verdaderamente de la acción humana y, para estos efectos, la Nueva Economía Institucional carece de los instrumentos teóricos y conceptuales necesarios. Esto se debe, a juicio del autor de este trabajo, a que se asume que el paso de las creencias y los valores al de la conformación de las instituciones informales se produce de forma casi automática, sin considerar ningún tipo de etapa intermedia, como si las instituciones tomaran forma instantáneamente. Pero entre el sistema de creencias (i.e. representación interna de la cultura) y 
las instituciones (i.e. su manifestación externa) no necesariamente hay un único camino o posibilidad. Por el contrario, la ruta de las creencias y los valores al de las instituciones está marcado por alternativas que responden a configuraciones sociales concretas y a los problemas puntuales que las personas deben resolver. Al respecto, Ann Swidler sugiere que la manera en la que la cultura influye en las acciones de los individuos no es a través de una relación directa entre los valores y la acción, sino proporcionando un conjunto de herramientas (hábitos, habilidades, estilos) que permiten a la persona desarrollar una estrategia de acción para desenvolverse en diferentes circunstancias y contextos (Swidler, 1986). Aplicando estos conceptos a la condición de pobreza, la autora hace referencia a investigaciones que señalan que los pobres y las clases medias comparten los mismos valores y aspiraciones, por lo que los comportamientos -y, por lo tanto, las instituciones informales- que caracterizan a unos y otros no pueden explicarse únicamente a partir de factores culturales. Swidler sintetiza esta idea de la siguiente manera:

Culture in this sense is more like a style or a set of skills and habits than a set of preferences or wants. If one asked a slum youth why he did not take steps to pursue a middle-class path to success (or indeed asked oneself why one did no pursue a different life direction) the answer might well be not "I don't want that life," but instead, "Who, me?" One can hardly pursue success in a world where the accepted skills, style and informal know-how are unfamiliar. One does better to look for a line of action for which one already has the cultural equipment. (Swidler, 1986, pág. 275)

Esta concepción de la cultura como un tool kit que permite diseñar estrategias de acción cuenta con el respaldo de programas de investigación tanto en la sociología contemporánea como en la psicología cognitiva (DiMaggio, 1997), y aporta una nueva interrogante en el intento de construir un marco teórico de la pobreza: ¿qué factores influyen en la estrategia de acción de las personas en condición de pobreza? En la Figura 2 se muestra el lugar que ocupa esta pregunta en la argumentación que se ha venido presentando.

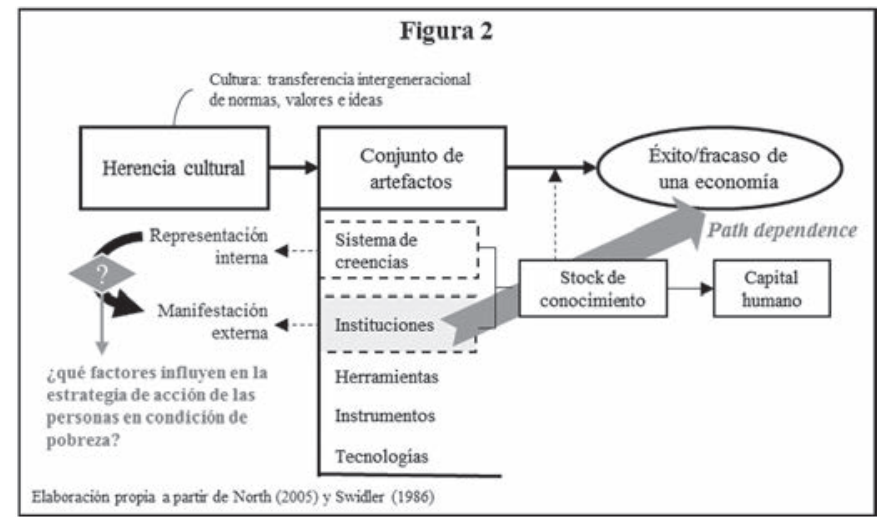


En la siguiente sección se intenta responder a la interrogante arriba planteada integrando al análisis conceptos y miradas propias de la Nueva Sociología Económica, particularmente las ideas de Mark Granovetter y Richard Swedberg.

\section{Las Redes Sociales: Origen del Marco Institucional}

Emphasis is placed on how economic activity comes to be coordinated by groups of people rather than carried out by isolated individuals. (Granovetter, 1992, pág. 3)

El punto de partida de esta sección es la crítica que se hiciera a la Nueva Economía Institucional por asumir que las instituciones son un producto directo de los factores culturales. Para comprender las dinámicas que configuran las instituciones se recurre a la Nueva Sociología Económica, aprovechando el interés que comparte con los economistas institucionales por incorporar el análisis de las instituciones sociales a la acción económica.

A manera de antecedentes, merece la pena recordar que hasta el siglo XIX no había mayor diferencia entre la sociología y la economía o, dicho de mejor manera, los problemas sociales y los económicos eran abordados de forma conjunta (Swedberg \& Granovetter, 1992). A medida ambas ciencias fueron tomando caminos diferentes, surgió una rama de la sociología que se denominó sociología económica y que tuvo en Max Weber a uno de sus principales exponentes. Desde sus inicios hasta la fecha, el propósito general de la sociología económica ha sido la aplicación de la perspectiva sociológica a los fenómenos económicos (Swedberg, 2006) y, concretamente, llenar el vacío que propició la corriente económica neoclásica al ignorar el rol de las instituciones en las actividades económicas de los individuos (Swedberg, 1997). A partir de mediados de la década de los ochenta del siglo XX se puede observar un renacer del interés de los sociólogos por integrar el estudio de las instituciones al de los problemas económicos, tal como en los años previos lo venía haciendo la Nueva Economía Institucional. A este resurgimiento se lo denomina Nueva Sociología Económica $^{12}$ (NSE), cuyo foco de atención es el desarrollo de una teoría sociológica de la construcción de las instituciones económicas (Granovetter, 1992). Una crítica que sociólogos de esta rama hacen a la economía institucional es el énfasis en la condición de eficiencia como tema central en el análisis de las instituciones ${ }^{13}$ (Swedberg \& Granovetter, 1992). Para ellos, las instituciones no surgen únicamente para reducir costos de transacción o para resolver "juegos

12. Para Swedberg (1997), el punto de partida y “manifiesto" de la Nueva Sociología Económica es el artículo Action and Social Structure: The Problem of Embeddedness de Mark Granovetter (1985). 13. Swedberg y Granovetter (1992) eximen a Douglass North de este anclaje tan profundo en la eficiencia y los costos de transacción. A su juicio, esto obedece al trabajo como historiador que North ha desarrollado. Se destaca aquí este punto debido a que North ha sido el autor principal utilizado en este trabajo para construir la primera parte del marco teórico. 
de coordinación social", sino que responden a otros condicionantes arraigados en el contexto social que se exponen más adelante.

En términos generales, la NSE se ha desarrollado en tres vertientes sociológicas: (1) la teoría de las redes, (2) la sociología cultural, y (3) la sociología organizacional (Swedberg, 1997). Conceptos y enfoques teóricos de las primeras dos son incorporados en este trabajo para complementar el marco teórico de la pobreza que se viene desarrollando.

\section{Bases conceptuales de la Nueva Sociología Económica}

Tomando como punto de partida el trabajo de Mark Granovetter (1985, 1992), puede decirse que la NSE pretende encontrar y desarrollar un espacio teórico que se sitúe entre la mirada "sobre socializadora"14 de la sociología tradicional, que considera al individuo como el resultado del contexto en el que participa, y el enfoque "infra socializador"15 de la economía neoclásica, que aísla al individuo en un mercado idealizado de competencia perfecta en el que las relaciones sociales son intrascendentes e innecesarias ${ }^{16}$. Para encontrar ese punto medio el autor recurre a tres premisas básicas de la sociología. En sus palabras,

(1) the pursuit of economic goals is normally accompanied by that of such non-economic ones as sociability, approval, status and power; (2) economic action (like all action) is socially situated and cannot be explained by individual motives alone; it is embedded in ongoing networks of personal relations rather than carried out by atomized actors ...; (3) economic institutions (like all institutions) do not arise automatically in some form made inevitable by external circumstances, but are 'socially constructed'. ${ }^{17}$ (Granovetter, 1992, pág. 4)

Para trasladar estas nociones a un nivel operativo que posibilite explicar de manera amplia la condición de pobreza, se desarrollan a continuación dos conceptos centrales del trabajo de Granovetter: las redes sociales y la idea de embeddedness. Aunque ambos se encuentran íntimamente relacionados, se abordan por separado para destacar sus aportes individuales a la comprensión de la pobreza.

14. En inglés, oversocialized. En Ritzer (1993) se traduce al español como orientación "normativa”.

15. En inglés, undersocialized. En Ritzer (1993) se traduce al español como orientación "atomista".

16. Aquí Granovetter critica lo que él considera es una versión extrema del individualismo económico en la economía. Esta crítica se asemeja a la de Boettke (2001) ya expuesta en este trabajo, en la que sugiere abandonar el individualismo atomístico para pasar a un individualismo institucional, que de ninguna manera implica dejar de lado las bases del individualismo metodológico.

17. Esta última idea está explícitamente inspirada en el trabajo de los sociólogos Peter Berger y Thomas Luckmann (1995), quienes exponen el rol de las instituciones en la construcción social de la realidad. De ahí que Granovetter hable en algunos pasajes acerca de la construcción social de la economía. 
Las redes sociales como punto de partida. En un trabajo previo a los de sociología económica citados en este documento, Granovetter explora la influencia de las redes personales -particularmente las de amistad- en la difusión de la información, las oportunidades de movilidad laboral y la organización de las comunidades (Granovetter, 1973). Estas redes se configuran a partir de vínculos fuertes y débiles, clasificación que obedece al tiempo que los involucrados pasan juntos, la intensidad emocional de la relación, el nivel de intimidad y confianza entre ellos, y los servicios recíprocos que caracterizan el vínculo. ${ }^{18}$ Por ejemplo, un colega con el que se convive diariamente en el trabajo representa un vínculo fuerte, mientras un cliente con el que alguna vez se cerró un negocio equivale a un vínculo débil.

Como es lógico asumir, los seres humanos tendemos a ser parte de grupos de vínculos fuertes, es decir, redes en las que todos los miembros tienen una relación cercana, e.g., los compañeros de la universidad, los integrantes del equipo de fútbol del barrio o las colegas del trabajo. Al interior de estos grupos o redes fluye información que todos los integrantes comparten: los temas de conversación y los intereses individuales son muy parecidos. De ahí que se integren en una red. Por otra parte, a lo largo de nuestra vida solemos establecer vínculos con individuos que no son parte de nuestras redes más cercanas, pero que las circunstancias nos han llevado a conocer. Es de suponer que estas personas a quienes nos une un vínculo débil son a su vez parte de redes de vínculos fuertes que manejan sus propios flujos de información.

Para Granovetter, las personas a quienes nos unen vínculos débiles representan puentes que nos conectan a otras redes, ampliando el conocimiento al que podemos acceder:

The fewer indirect contacts one has the more encapsulated he will be in terms of knowledge of the world beyond his own friendship circle; thus, bridging weak ties (and the consequent indirect contacts) are important in both ways... [T] hose to whom we are weakly tied are more likely to move in circles different from our own and will thus have access to information different from that which we receive. (1973, pág. 1371)

Esto tiene un impacto grande en la difusión de la información, la movilidad laboral y la organización de las comunidades, entre otras situaciones. ${ }^{19}$ Especial

18. Ninguno de estos factores puede definirse de manera "objetiva"; se trata únicamente de parámetros que permite concluir sobre la fuerza o debilidad del vínculo. En la evidencia empírica presentada por Granovetter (1973) se utiliza como principal parámetro el tiempo relativo que las personas permanecen juntas. Estos estudios no pretenden ofrecer una medida objetiva, sino más bien ilustrar el fenómeno.

19. Los aportes de Granovetter en esta área representan un punto de partida para el desarrollo contemporáneo de la ciencia de las redes (Watts, 2004). 
atención para el tema que nos ocupa tienen el segundo y tercero de estos ámbitos. En el caso de la movilidad laboral -partiendo del supuesto que para abandonar la condición de pobreza una persona debe estar en condiciones de generar un flujo de ingresos suficientes a través del libre intercambio de su trabajo por dinero $^{20}$ - resulta evidente que mientras más vínculos débiles (puentes) pueda establecer una persona en situación de pobreza con individuos que estén en condición de ofrecerle o conseguirle un trabajo mejor remunerado, mayores son sus posibilidades de salir de la pobreza. Por el contrario, una persona que nace, crece y se desarrolla exclusivamente en el ámbito de una comunidad en situación de pobreza, sin puentes con comunidades más prósperas en las que hay mayor acceso a educación y trabajo, tiene mayores probabilidades de permanecer en la pobreza.

Para el caso de la organización de las comunidades la situación es muy similar. Sin la presencia de vínculos débiles que conecten a miembros de subgrupos con otras agrupaciones, la organización y desarrollo de la comunidad se verá limitada. Granovetter señala esta situación claramente: "Imagine, to begin with, a community completely partitioned into cliques, such that each person is tied to every other in his clique and to none outside. Community organization would be severely inhibited" (Granovetter, 1973, pág. 1373). Este tipo de aislamiento es el que puede observarse con facilidad en los guetos, cuyos habitantes se relacionan en grupos pequeños con un mínimo contacto con otras agrupaciones dentro del mismo territorio, ya no se diga con personas pertenecientes a entornos en los que podrían encontrar oportunidades laborales adecuadas para generar un flujo de ingresos que les permita salir de la pobreza. En síntesis, los vínculos débiles "evitan el aislamiento y permiten a los individuos estar más integrados en la sociedad” (Ritzer, 1993, pág. 449).

Al respecto de los puntos anteriores, la evidencia empírica que presentan Narayan et al. (2000) sugiere que cuando las personas de las comunidades pobres se encuentran desvinculadas de otras personas pertenecientes a comunidades de similares características, resulta difícil llegar al nivel de organización necesario en torno a temas concretos para alcanzar el progreso social. De hecho, se observa que los grupos de los ricos son más cohesionados que los de los pobres. A la luz de las ideas desarrolladas por Granovetter puede afirmarse que contar con un número significativo ${ }^{21}$ de vínculos débiles es condición sine qua non para salir de la pobreza. Dicho a la inversa, una persona viviendo en condición de pobreza que no es capaz de establecer vínculos (débiles) con otras redes sociales, permanecerá en esa situación por tiempo indefinido. Estas afirmaciones nos

20. Ciertamente esta persona también podría emprender un negocio propio y no depender de la venta directa de su mano de obra, sin embargo, para simplificar la argumentación ésta se ilustrará con casos en los que se ingresa al mercado laboral.

21. No es posible establecer un número "adecuado" u "objetivo" de vínculos débiles necesarios. El punto es señalar la importancia de los puentes en la generación de oportunidades de desarrollo. El calificativo significativo podría significar uno solo que resulte ser sumamente relevante. 
permiten retomar la mirada de la pobreza como un problema de coordinación, tal como se expuso en la sección III. Al no poder relacionarse y coordinarse -a través de los vínculos personales débiles- con otros grupos de individuos que manejan información diferente, las personas en situación de pobreza difícilmente prosperan económicamente.

La condición de embeddedness ${ }^{22}$ de la acción económica. El concepto más reconocido e importante de la NSE es, precisamente, embeddedness (Swedberg, 2006). A través de él, Mark Granovetter propuso una interpretación de la acción económica alejada del atomismo de la economía neoclásica, pero sin caer en la sobre socialización que caracteriza a algunas corrientes sociológicas que también han intentado abordar el problema económico (Granovetter 1985, 1992). Para él, las decisiones y actividades de orden económico que los individuos emprenden están empotradas (embedded) en las redes de relaciones interpersonales a las que pertenecen. Incluso las instituciones surgen a partir de estas redes y se desarrollan de acuerdo a sus características. Al explicar este último punto, Granovetter se distancia de los economistas institucionales que conciben a las instituciones eminentemente como respuestas a problemas de eficiencia en los costos de transacción. Estas ideas se sintetizan en el siguiente párrafo:

But economic institutions do not emerge automatically in response to economic needs. Rather, they are constructed by individuals whose action is both facilitated and constrained by the structure and resources available in social networks in which they are embedded. (Granovetter, 1992, pág. 7).

Aunque la mayor parte de su argumentación se ejemplifica mediante evidencia empírica acerca de la organización de firmas y grupos empresariales, la propuesta de Granovetter de entender la acción económica desde el sistema de redes sociales a las que los individuos tienen acceso puede aplicarse al estudio de la pobreza, siempre y cuando ésta sea concebida como un problema de coordinación, tal como se ha sostenido en este trabajo ${ }^{23}$. De esta manera, la ausencia de un proceso de coordinación que permita a los individuos en condición de pobreza intercambiar su capacidad laboral y productiva con quienes están en condiciones de pagarla, puede encontrar una explicación en la calidad ${ }^{24}$ y

22. Gómez Fonseca (2004) discute las posibles traducciones del concepto embeddedness -con la intención que lo usa Granovetter-al español. Para él, las acepciones más aceptables son integración, soporte, infiltración, apropiación, empotrar e incrustar, aunque concluye sugiriendo no circunscribirse a una sola. En Ritzer (2003) se traduce como una acción "engastada". Ante tal diversidad de alternativas y, para no desviar el foco de atención de este trabajo, se utiliza el término en inglés, según corresponda (embedded o embeddedness).

23. La mirada tecnócrata de la pobreza no da lugar a este tipo de consideraciones, en la medida en que se centra en la concentración ("desigual” e "injusta”) del ingreso más que en la capacidad de cada persona por generar su propio ingreso.

24. Aquí el término calidad no se utiliza en referencia a las cualidades humanas de las personas con quienes se interactúa, sino a la capacidad de éstas para establecer relaciones de intercambio económico. 
alcance (definido por sus vínculos débiles) de las redes sociales a las que se pertenece. Redes que, eventualmente, dan lugar a las instituciones económicas en las que los individuos participan. Así, para Granovetter,

stable economic institutions begin as accretions of activity patterns around social networks. Their structures reflects that of the networks, and even when those are no longer in place, the institutions take on a life of their own that limits the forms future ones can take; they become 'locked in'. (1992, pág. 9)

Para ejemplificar la relación causal entre las redes sociales y las instituciones económicas, puede tomarse el caso de algunas regiones de África en las que los vínculos familiares son tan fuertes que desincentivan el ahorro y, por lo tanto, inhiben el desarrollo económico. Esto se da debido a que cada miembro de la familia tiene la "obligación" de compartir con el resto todos sus bienes y sus ganancias (Narayan et al., 2000).

\section{Complementando la mirada institucional}

En la Figura 2 se enfatizaba y criticaba la ausencia -en la mirada de la Nueva Economía Institucional, particularmente en el caso de North- de un vínculo entre el mundo de las ideas y la conformación de las instituciones informales. Ese vacío puede ser llenado por los conceptos de redes sociales y embeddedness expuestos en los párrafos anteriores. En la Figura 3 se presenta esta incorporación.

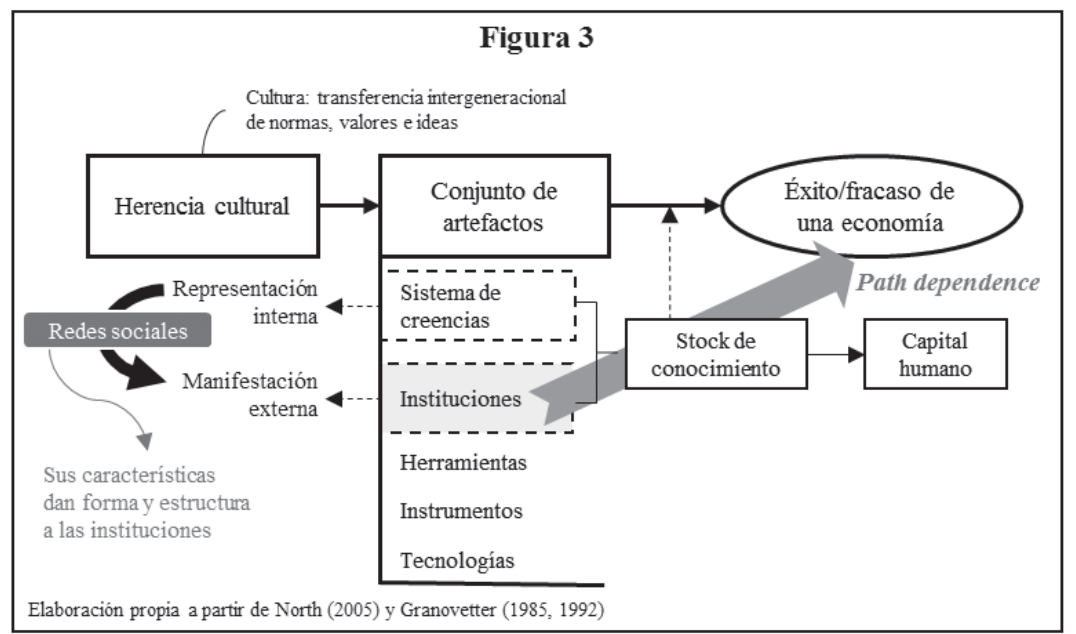

Estos aportes de la Nueva Sociología Económica, además, refuerzan y complementan tres ideas que ya se expusieron en este trabajo: (1) la necesidad de aplicar el teorema de la regresión de Mises para analizar el cambio institucional (Boettke, 2001), (2) la capacidad de adherencia de las indigenously introduced endogenous institutions (IEN) y su utilidad al momento de aplicar el 
teorema de la regresión (Boettke et al, 2008); y (3) el peligro de aplicar una política de "pizarra en blanco" para diseñar soluciones técnicas al problema de la pobreza (Easterly, 2013). En el caso de la primera, la regresión conducirá al sistema de redes sociales en el que se desenvuelven los individuos y al tipo de acción económica que se genera a partir de estas redes. En la segunda, el componente endógeno es el resultado de lo que un determiando sistema de redes es capaz de producir. Y en el caso de la política de "pizarra en blanco", su fracaso resulta de ignorar las características y la importancia de las relaciones entre los individuos de la región que se pretende "rescatar" de la pobreza.

\section{Dando Forma a un Modelo de Análisis de la Pobreza}

[L]a realidad es que aún podemos leer afirmaciones en el sentido de que las teorías científicas son probadas por los hechos. ¿A qué se debe esa obstinada oposición a la lógica elemental? (Lakatos, 1983, pág. 11)

Con el propósito organizar las ideas y conceptos expuestos hasta este punto, se recurre a algunas de las categorías que Imre Lakatos propone como parte de su metodología para programa de investigación científica ${ }^{25}$ (Lakatos, 1983). Como se ha venido sugiriendo en este trabajo, la pobreza no puede verse simplemente como "la otra cara de la moneda" de la riqueza. Los marcos institucionales en los que se sostiene la actividad económica de quienes viven en condición de escasez material y que les impiden coordinarse eficientemente con otros sujetos, tienen dinámicas y particularidades propias. Por este motivo se sostiene aquí que la pobreza, en tanto resultado de un proceso de coordinación deficiente, merece un modelo de análisis particular.

En cuanto a la metodología de Lakatos, se retoman dos de sus partes: el núcleo firme y el cinturón protector de hipótesis auxiliares. La heurística se ha dejado de lado debido a que requiere un desarrollo metodológico y empírico que excede a los alcances de este trabajo.

Para el problema que se investiga aquí, el núcleo firme está conformado por el problema de coordinación que, como se ha señalado antes, es uno de los pilares del núcleo general de la Escuela Austriaca de Economía (Rizzo, 1982). Tomar al proceso de coordinación social como el eje en el que se sustenta el análisis de la pobreza implica aceptar la condición de conocimiento disperso y subjetivo, así como el reconocimiento de la capacidad de las personas por aprender mutuamente (señalada por Hayek) y el surgimiento de una especulación empresarial para coordinar la satisfacción de las necesidades de los individuos (destacado por Mises)

25. En este trabajo no se pretende desarrollar una construcción rigurosa de un programa de investigación en el sentido que Lakatos lo define. Como se explica más adelante, esta investigación representa las bases para su posterior desarrollo. 
(Kirzner, 1997). Las implicaciones para el análisis de la pobreza se sintetizan en la siguiente afirmación: una persona se mantiene en condición de pobreza cuando no es capaz de coordinar con otros un intercambio productivo, que le permita satisfacer sus necesidades materiales más elementales.

El cinturón protector de este núcleo está compuesto por tres hipótesis auxiliares. La primera se origina en la Nueva Economía Institucional y si sintetiza así: el marco institucional que facilita o dificulta la coordinación entre los individuos se sostiene en un conjunto de instituciones informales, basadas en las creencias y herencia cultural de los participantes. Esto implica que cualquier formalización institucional -a través de normas o leyes-debe derivarse de las raíces culturales. La segunda hipótesis auxiliar se extrae de la Nueva Sociología Económica (NSE) y se vincula fuertemente a la primera: las instituciones informales se configuran de acuerdo a las redes sociales a las que pertenecen los individuos. De esta forma, redes muy cerradas que se caracterizan por la desconfianza hacia otras redes, generarán instituciones informales que acentuarán esa desconfianza, impidiendo la coordinación más allá del grupo social más próximo. La tercera hipótesis también se origina en la NSE y tiene que ver con la importancia de los vínculos débiles a la que hacía referencia Granovetter: las personas que viven en situación de pobreza tienen pocos vínculos débiles que los conecten a redes en las que podrían coordinar de manera más eficiente y efectiva su capacidad productiva. Dicho en otros términos, las comunidades de personas pobres se encuentran socialmente aisladas, haciéndose evidente la distinción entre "ellos" y "nosotros" de la que hablan Coyne y Mathers (2011).

\section{Poniendo a prueba la relación entre los elementos del modelo de análisis}

A pesar que en este trabajo no se ha pretendido en ningún momento obtener y sistematizar evidencia empírica, se hace uso de un ejemplo práctico para poner a prueba la capacidad de explicar la condición de pobreza del núcleo firme e hipótesis auxiliares. La intención es únicamente ilustrar el valor teórico del modelo, no validarlo empíricamente.

Se trata de la historia de vida Félix, ${ }^{26}$ un salvadoreño de treinta y siete años de edad quien, en menos de siete años, pasó de vivir en la comunidad en la que nació y creció en un ambiente de pobreza, a comprar una casa en una zona de clase media, en la que ahora habita con su esposa y sus cuatro hijos. Su transición de trabajar de manera informal en una discoteca móvil a desempeñarse como Supervisor Regional de Eventos en uno de las empresas de publicidad más importantes de Centro América - sin haber cursado estudios universitarios o

26. La historia que se utiliza en el ejemplo es real. Para efectos de presentarla, se realizó una entrevista en profundidad al sujeto, obteniendo su consentimiento para publicarla en este trabajo. Se empleó este método poco común en análisis económicos siguiendo la sugerencia de Boettke (2001) ya expuesta en páginas anteriores: es necesario dotar al análisis económico de una perspectiva multidisciplinario, utilizando, por ejemplo, los aportes de la etnografía. 
especializados- puede explicarse utilizando el modelo de análisis propuesto. Para ello se parte de las hipótesis auxiliares para luego concluir en el núcleo firme.

Un marco institucional (informal) que dificulta la coordinación. Al igual que buena parte de los jóvenes que viven en su comunidad de origen -localizada en la ciudad de San Salvador- Félix tuvo su primer hijo cuando aún era muy joven. Él lo adjudica al tiempo libre del que todos disponen durante el día, mismo que utilizan para deambular por las calles o jugar béisbol en el estadio contiguo a la comunidad. Esta situación hace que la planificación de una ruta de vida en la que los estudios sean un elemento central quede fuera del panorama. Desde muy jóvenes, aquellos que asumen la responsabilidad de su paternidad deben trabajar para procurar el sustento de su familia. Lo normal es aprender empíricamente algún oficio y practicarlo de la forma que sea. Desarrollarse profesionalmente no es una espiración que se persiga con ahínco. El denominador común es vivir la vida día a día. Al carecer de un lugar en el que habitar con su pareja, Félix construyó su vida familiar en la pequeña casa de su madre. Hasta hace poco, vivían los siete (él, su esposa, cuatro hijos y su madre) en un espacio de unos ocho por ocho metros.

Estas instituciones culturales en las que se sustenta la actividad económica de los jóvenes de la zona -ausencia de planificación, responsabilidad familiar temprana, informalidad en el trabajo como algo natural- indudablemente tiene un impacto negativo en el tipo de coordinación que se puede establecer con otras personas.

Las características de las redes sociales configuran las instituciones. Las instituciones culturales en la que se sostenía la actividad económica de Félix son el resultado de las redes sociales en las que se desenvolvía. Como ya se mencionó, su comunidad está ubicada junto a un estadio de béisbol. Esto hace que el interés de los jóvenes de la zona por ese deporte sea grande, al punto que la gran mayoría lo practica diariamente. Como un dato al margen, en El Salvador el béisbol no cuenta con una estructura profesional que permita crecer económicamente a quienes lo practican. Son muy pocas las personas de la zona que, sin haber emigrado a otro país, han logrado abandonar la condición de pobreza. Esto hace que no existen "ejemplos a seguir" para los más jóvenes.

El tiempo dedicado al deporte -sin llegar a practicarlo profesionalmente-, la falta de referentes de superación en la zona y poco contacto con otros sectores de la población son posibles explicaciones a las instituciones arriba expuestas.

La fuerza de los vínculos débiles. La situación de confinamiento en la que viven la mayoría de las personas de comunidades como la de Félix provoca que los vínculos con otras redes sean escasos. En el mejor de los casos, se relacionan con otros grupos en situación económica similar, por ejemplo, en la escuela o en torneos de béisbol amateur. Esta falta de contacto con otras redes perpetúa 
las características de la propia e imposibilita el cambio institucional necesario para transformar la actividad económica de la zona. Sin embargo, algunos de ellos logran establecer estas conexiones, aunque no siempre son aprovechadas. Aquí es donde la historia de Félix toma un rumbo diferente.

La práctica del deporte desde la juventud permite a algunos participar en ligas de softbol amateur para adultos en las que juegan personas que tienen una situación económica más próspera. Jóvenes como Félix participan como refuerzos en equipos que son financiados por estas personas. Esto genera grandes oportunidades para establecer vínculos débiles y tender puentes con otras redes. Incluso, algunos de los jóvenes refuerzos reciben un pago por cada partido jugando. De hecho, muchos lo ven como una fuente de ingresos, alcanzando el grado de institución informal: "solo formo parte de un equipo si me pagan”. En el caso de Félix, la situación nunca fue así. Él jugaba para uno de estos equipos solo por diversión, sin exigir dinero por hacerlo. Esto llamó la atención de uno de los integrantes del equipo, que es uno de los principales directores de la empresa en la que Félix se ha desarrollado. Al ver que su comportamiento era diferente le ofreció un trabajo sencillo, que no requería de preparación previa. Poco a poco, Félix ha ido ascendiendo hasta ocupar un cargo que le permite llevar una vida más holgada: ya tiene una casa propia, sus hijas asisten a un colegio privado y luego ingresarán a la universidad. Aunque él considera que aún no ha salido del todo de la condición de pobreza, lo cierto es que hoy está en condiciones de planificar su futuro.

De la descoordinación a la coordinación. Como se ha venido sosteniendo, la pobreza resulta de la incapacidad de coordinarse eficientemente. En el caso de los jóvenes de la comunidad en la que vivió nuestro sujeto, la falta de una coordinación eficiente es evidente. En la medida en que no consigan intercambiar su fuerza laboral de manera formal y consistente en el tiempo resulta improbable que abandonen la condición de pobreza en la que siempre han vivido. Esta falta de coordinación es el resultado de marcos institucionales que la impiden, sustentados en redes sociales estrechas.

En el caso de Félix, quien ahora está en condiciones de coordinar su actividad económica con personas dispuestas a pagarle un buen salario, el punto de inflexión estuvo en la ampliación de su red social. Un vínculo débil -alguien a quien solo veía una vez por semana en un juego de softbol- le permitió acceder a un nueva red social en la que aprendió cómo desenvolverse en un ambiente de oficina. Entrar a esta red le permitió formar parte de instituciones que le eran ajenas, e.g. trabajar en base a metas o planificar su vida personal de acuerdo a un flujo de ingresos estable. 


\section{Conclusiones y Consideraciones Finales}

La condición de pobreza en la que aún viven cientos de millones de personas es un tema prioritario en la agenda política y académica. Sin embargo, su abordaje es cada vez más abstracto y técnico. Los debates se centran en conceptos relativos como desigualdad o injusticia distributiva, o en estadísticas y cálculos fríos. Las soluciones, en consecuencia, suelen ser técnicas y de aplicación estandarizada. El presente trabajo se desarrolló con el propósito de ofrecer una respuesta a la siguiente interrogante: ¿de qué manera puede estructurarse un programa de investigación que permita estudiar de manera sistemática y progresiva, desde diversas áreas de conocimiento, la condición de pobreza en la que aún viven cientos de millones de personas? Para contestarla se partió de la premisa que para alcanzar una comprensión más próxima a la realidad de quienes viven en la pobreza es necesario construir un marco teórico que tenga su punto de partida en las personas y no en las cifras. Asimismo, la complejidad del fenómeno -tal como sucede con todos los fenómenos sociales- exige que el modelo de análisis integre diversas disciplinas y enfoques.

El marco teórico que se propone tiene como eje o núcleo fuerte el problema de coordinación, tal como es conceptualizado en la tradición de la Escuela Austriaca de Economía. Lo que se sugiere es que la pobreza es el resultado de la ausencia de un proceso de coordinación adecuado para que se produzca un intercambio de recursos efectivo. Para explicar este problema de coordinación a partir de la cotidianidad de los individuos, respetando sus creencias y herencia cultural, se formularon tres hipótesis auxiliares que permiten explicar la presencia o ausencia del proceso de coordinación necesario para abandonar la pobreza: (1) la influencia de las instituciones informales como facilitadoras u obstaculizadoras de la coordinación (esta idea se retoma de la Nueva Economía Institucional); (2) la importancia de las redes sociales en la configuración de esas instituciones informales; y (3) el aislamiento en el que se desenvuelven las comunidades de personas en condición de pobreza o, dicho de otra manera, su falta de vínculos débiles que los conecten con otras redes de personas (estas últimas dos hipótesis basadas en conceptos claves de la Nueva Economía Sociológica).

Es importante notar que esta aproximación al tema únicamente es posible si se produce un cambio de paradigma en cuanto al análisis y abordaje de la condición de pobreza: se debe transitar de la idea de asignación de recursos al enfoque en el intercambio de recursos.

\section{¿Dónde queda el Estado en esta discusión?}

Resulta difícil hablar de pobreza sin que se plantee cuál debe ser el rol del Estado en los esfuerzos por combatirla. A lo largo de este trabajo se ha omitido de manera intencional la figura del Estado para concentrar la atención en las 
dinámicas económicas, culturales y sociales que son independientes del papel que éste desempeñe. La coordinación social, las instituciones informales y las redes sociales son fenómenos ajenos al Estado, es decir, suceden tanto en presencia como en ausencia de éste.

En cuanto al rol que pueda desempeñar, del modelo de análisis propuesto se infieren los siguientes: (1) propiciar los espacios de interacción adecuados para ampliar el tejido social mediante la formación de vínculos débiles; (2) respetar la herencia cultural al momento de formalizar las instituciones informales; y (3) garantizar el marco institucional necesario para una coordinación social eficiente. Las fórmulas del estilo de "pizarra en blanco" deben descartarse, pues son incapaces de adaptarse a la naturaleza de las redes sociales ya existentes. Numeroso ejemplos de comunidades pobres que han resuelto algunos de sus problemas inmediatos mediante iniciativas propias de cooperación como las asociaciones de ahorro y préstamo, sociedades funerarias y organizaciones de mujeres empresarias, ${ }^{27}$ por mencionar algunas, son muestra de cómo las personas son capaces de organizarse por ellas mismas.

\section{Caminos de investigación}

La principal línea de investigación que se deriva de este trabajo es la formalización de un programa de investigación científica en el sentido de Lakatos. Para ello, debe profundizarse en los elementos del núcleo firme y desarrollar nuevas hipótesis auxiliares que lo protejan. También es necesario desarrollar una heurística positiva que sugiera el camino de desarrollo.

Adicionalmente, y en apoyo a lo anterior, debe construirse evidencia empírica que refuerce el modelo, combinando metodologías tradicionales de la economía con métodos etnográficos propios del estudio de la cultura.

\section{Bibliografía}

Baumol, W. J., \& Blinder, A. S. (1994). Economics. Principles and Policy (6 Ed.). Orlando: The Dryden Press.

Berger, P., \& Luckmann, T. (1995). La construcción social de la realidad. Buenos Aires: Amorrortu.

Boettke, P. J. (1990). The Theory of Spontaneous Order and Cultural Evolution in the Social Theory of F. A. Hayek. Cultural Dynamics, 3(1), 61-83. doi:10.117/092137409000300105

Boettke, P. J. (2001). Why culture matters. En P. J. Boettke, Calculation and Coordination. Essays on socialism and transitional political economy (págs. 248-265). London: Routledge. 
Boettke, P. J., Coyne, C. J., \& Leeson, P. T. (2008). Institutional Stickiness and the New Development Economics. American Journal of Economics and Sociology, 67(2), 331-358.

Coyne, C. J. (2010). Economics as the study of coordination and exchange. En P. J. Boettke, Handbook on Contemporary Austrian Economics (págs. 14-29). Cheltenham, UK: Edward Elgar.

Coyne, C. J., \& Boettke, P. J. (2006). The Role of the Economist in Economic Development. The Quarterly Journal of Austrian Economics, 9(2), 47-68.

Coyne, C. J., \& Mathers, R. L. (2011). Rituals: An economic interpretation. Journal of Economic Behavior \& Organization, 78, 74-84. doi:10.1016/j.jebo.2010.12009

de Soto, H. (2001). El Misterio del Capital. México, D.F.: Diana.

DiMaggio, P. (1997). Culture and Cognition. Annual Review of Sociology, 23, 263-287. doi:10.1146/annurev.soc.23.1.263

Dollar, D., \& Kraay, A. (2004). Trade, Growth, and Poverty. The Economic Journal, 114(493), 22-49.

Easterly, W. (2013). The Tyranny of Experts. Economists, Dictators, and the Forgotten Rights of the Poor (Edición Kindle). New York: Basic Books.

Fukuyama, F. (1996). Trust. The Social Virtues and the Creation of Prosperity (versión Kindle). New York: Free Press Paperback.

Gilles, C. (23 de mayo de 2014). Financial Times. Obtenido de Piketty findings undercut by errors: http://www.ft.com/cms/s/2/e1f343ca-e281-11e3-89fd-00144feabdc0. html\#axzz345Jkvohw

Gómez Fonseca, M. Á. (2004). Reflexiones sobre el concepto de embeddedness. Polis, 2, $145-164$.

Granovetter, M. (1973). The Strength of Weak Ties. American Journal of Sociology, 78(6), $1360-1380$.

Granovetter, M. (1985). Economic Action and Social Structure: The Problem of Embeddedness. The American Journal of Sociology, 91(3), 481-510.

Granovetter, M. (1992). Economic Institutions as Social Constructions: A Framework for Analysis. Acta Sociologica, 35, 3-11.

Guiso, L., Sapienza, P., \& Zingales, L. (2006). Does Culture Affect Economic Outcomes? The Journal of Economic Perspectives, 20(2), 23-48.

Hayek, F. A. (1945). The Use of Knowledge in Society. The American Economic Review, 35(4), 519-530.

Hayek, F. A. (1948a). Economics and Knowledge. En Individualism and Economic Order (págs. 33-56). Chicago: The University of Chicago Press.

Hayek, F. A. (1948b). The Facts of the Social Sciences. En Individualism and Economic Order (págs. 57-76). Chicago: The University of Chicago Press.

Hayek, F. A. (1978). The Constitution of Liberty. Chicago: The University of Chicago Press. Hazlitt, H. (1996). The Conquest of Poverty. Irvington-on-Hudson: The Foundation for Economic Education.

Hodgson, G. M. (2006). What Are Institutitons? Journal of Economic Issues, XL(1), 1-25.

Hofstede, G. (1999). Culturas y Organizaciones. El Software Mental. Madrid: Alianza Editorial.

Hofstede, G., \& Harris Bond, M. (1988). The Confucius Connection: From Cultural Roots 
To Economic Growth. Organizational Dynamics, 16(4), 5-21.

Horwitz, S. (2000). From The Sensory Order to the Liberal Order: Hayek's Non-rationalist Liberalism. Review of Austrian Economics, 13, 23-40.

Kirzner, I. M. (1997). How Markets Work. Londres: The Institute of Economic Affairs.

Krause, M. (2013). El foro y el bazar: Economía, instituciones y políticas públicas (Edición Kindle). Guatemala: Universidad Francisco Marroquín.

Krugman, P. (24 de mayo de 2014). The New York Times. Obtenido de Is Piketty All Wrong?: http://krugman.blogs.nytimes.com/2014/05/24/is-piketty-all-wrong/

Lachmann, L. M. (1977). Capital, Expectations, and the Market Process. Kansas City: Sheed Andrews and McMeel.

Lakatos, I. (1983). La metodología de los programas de investigación científica. Madrid: Alianza Editorial.

Mandel, M. (2009). Economics. The Basics. New York: McGraw-Hill.

Mankiw, N. G. (2002). Principios de Economía. Madrid: McGraw-Hill.

Mason, P. (26 de mayo de 2014). The Guardian. Obtenido de Thomas Piketty's real chalenge was to the FT's Rolex types: http://www.theguardian.com/ commentisfree/2014/may/26/thomas-piketty-economist-ft-attack-risinginequality-bling

Mises, L. v. ([1949] 1996). Human Action. A Treatise On Economics (4 Ed.). San Francisco: Fox \& Wilkes.

Narayan, D., Patel, R., Schafft, K., Rademacher, A., \& Koch-Schulte, S. (2000). Voices of the Poor. Can Anyone Hear Us? New York: Oxford University Press for the World Bank.

Niemietz, K. (2011). A New Understanding of Poverty. Poverty Measurement and Policy Implications. Londres: The Institute of Economic Affairs.

North, D. C. (1990). Institutions, Institutional and Economic Performance (Edición Kindle). Cambridge: Cambridge University Press.

North, D. C. (1991). Institutions. The Journal of Economic Perspectives, 5(1), 97-112.

North, D. C. (2005). Understanding the Process of Economic Change (Edición Kindle). Princeton: Princeton University Press.

North, D. C. (2012). La Nueva Economía Institucional. En A. Ravier, Lecturas de Historia del Pensamiento Económico (versión Kindle). Madrid: Unión Editorial.

O'Driscoll, J. G. (1977). Economic as a Coordination Problem. The Contributions of Friedrich A. Hayek. Kasnsas City: Sheed Andrews and McMeel.

Parkin, M. (2004). Economía (6 Ed.). Naucalpán de Juárez: Pearson.

PNUD. (2013a). Informe sobre Desarrollo Humano 2013. El ascenso del Sur: Progreso humano en un mundo diverso. Nueva York: PNUD.

PNUD. (2013b). Informe sobre Desarrollo Humano El Salvador 2013. Imaginar un nuevo país. Hacerlo posible. Resumen. San Salvador: Programa de las Naciones Unidas para el Desarrollo.

Ravallion, M. (2003). The Debate on Globalization, Poverty and Inequality: Why Measurement Matters. International Affairs, 79(4), 739-753.

Ritzer, G. (1993). Teoría Sociológica Contemporánea. Madrid: McGraw-Hill.

Rizzo, M. J. (1982). Mises and Lakatos: A Reformulation of Austrian Methodology. En I. Kirzner, Method, Process, and Austrian Economics. Essays in Honor of Ludwig von Mises (págs. 53-72). Lexington, Massachusetts: D.C. Heath and Company. 
Rothbard, M. N. ([1962] 2001). Man, Economy, and State. Auburn: The Ludwig von Mises Institute.

Samuelson, P. A., \& Nordhaus, W. D. (1999). Economía (16 Ed.). Madrid: McGraw-Hill.

Seidler, V. (2014). When do institutional transfers work? The relation between institutions, culture and the transplant effect: the case of Borno in north-eastern Nigeria. Journal of Institutional Economics, 1-27. doi:10.1017/S1744137414000046

Stiglitz, J. E. (2006). Making Globalization Work. New York: W. W. Norton \& Company.

Swedberg, R. (1997). New Economic Sociology: What Has Been Accomplished, What Is Ahead? Acta Sociologica, 40, 161-182.

Swedberg, R. (2006). The Toolkit of Economic Sociology. SOCIUS Working Papers.

Swedberg, R., \& Granovetter, M. (1992). Introduction. En M. Granovetter, \& R. Swedberg, The Sociology of Economic Life (págs. 1-26). Boulder, EEUU: Westview Press.

Swidler, A. (1986). Culture in Action: Symbols and Strategies. American Sociological Review, 51(2), 273-286.

Tabellini, G. (2010). Culture and Institutions: Economic Development in the Regions of Europe. Journal of the European Economic Association, 8(4), 677-716.

The World Bank. (2014). Highlights: World Development Indicators 2014. Washington DC: The World Bank.

Tucker, I. B. (2002). Fundamentos de Economía ( $3^{\circ}$ Ed.). México, DF: Thomson Learning. Watts, D. J. (2004). Six Degrees. The Science of a Connected Age. Londres: Vintage.

Williamson, C. R. (2009). Informal Institutions Rule: Institutional Arrangements and Economic Performance. Public Choice, 139(3/4), 371-387.

Williamson, C. R., \& Mathers, R. L. (2011). Economic freedom, culture, and growth. Public Choice, 148, 313-335. doi:10.1007/s11127-010-9656-Z 